\title{
Frequency Dependence of Intrinsic Stress and Birefringence Tensor of Bead/Spring Model of Polymer Solutions
}

\author{
A. Peterlin \\ Institute for Materials Research, National Bureau of Standards, Washington, D.C. 20234 \\ and \\ J. T. Fong \\ Institute for Basic Standards, National Bureau of Standards, Washington, D.C. 20234 \\ (November 22, 1976)

\begin{abstract}
The recently obtained complete solution of the simultaneous diagonalization of matrices $\mathbf{H} \mathbf{A}$ and $\mathbf{H}$ in the hydrodynamic diffusion equation has basically changed the diagonal values $\nu_{p}$ of the symmetric matrix $\mathbf{H}$ of hydrodynamic interaction between all the beads of the elastic random coil model of the isolated macromolecule in solution. Since these values enter explicitly the expressions for the intrinsic stress and refractive index tensor in an alternating flow field if based on the concept of internal viscosity of the model one had to recalculate all values obtained formerly by using the then generally accepted erroneous set of $\nu_{p}$ data. The new $\nu_{p}$ equal unity independent of $p$ while the old values were larger than 1 for small $p$ and smaller for large $p$. Hence their too large contributions in the former range are partially compensated by their too small contributions in the latter region. As a consequence in the whole range investigated, between 3 and 300 chain links, the differences in rheological and rheooptical effects are relatively small, up to a factor of 2 , although at higher link number the differences tend to grow with the logarithm of this number.
\end{abstract}

Key words: Bead-spring model; eigenvalues; frequency response; intrinsic optical tensor; intrinsic stress tensor; polymer solution.

\section{Introduction}

The correct simultaneous diagonalization $[1]^{1}$ of $\mathbf{H} \mathbf{A}$ and $\mathbf{H}$ matrices in Zimm's hydrodynamic equation [2] for the ideally flexible necklace model of randomly coiled isolated linear macromolecule in laminar flow makes possible a more adequate calculation of intrinsic stress tensor in all those cases where the coil is not yet noticeably deformed by the flow. Such a zero gradient case includes the frequency dependence of viscosity [2], shear modulus, shear birefringence, normal stress difference, and acoustic birefringence but not the gradient dependence of these effects.

The main change introduced by the new solution as compared with the older incomplete solutions [3-8] is not in the eigenvalues $\lambda_{p}$ of $\mathbf{H} \mathbf{A}$

$$
\mathbf{Q}^{-1} \mathrm{H} \mathbf{A} \mathbf{Q}=\mathbf{\Lambda}
$$

which were already calculated correctly in recent papers [6-8] and even tabulated for $Z$ between 1 and 15 and $h^{*}=$ $(3 / \pi)^{1 / 2} a_{h} / b_{0}=.01, .1, .2, .3,[6]$ and for $Z=250, h^{*}=$ .3 and $Z=300, h^{*}=0.4[1]$. Here $a_{h}$ is the hydrodynamic radius of the bead, $Z+1$ is the number of beads, $Z$ is the number and $b_{0}$ the root mean square length of the links. The difference is in the diagonal elements $\nu_{p}$ of the tensor

$$
\mathbf{Q}^{-1} \mathbf{H} \mathbf{Q}^{-1 T}=\mathbf{N}=\mathbf{1}
$$

which turns out to be the unity tensor with all $\nu_{p}=1$. This makes the diagonal elements of

$$
\mathbf{M}=\mathbf{Q}^{T} \mathbf{A} \mathbf{Q}=\mathbf{\Lambda} / \mathbf{N}=\boldsymbol{\Lambda}
$$

agree with those of $\boldsymbol{\Lambda}$, i.e. $\mu_{p}=\lambda_{p}$. Here $\mathbf{Q}$ is the transformation matrix of the original $3(Z+1)$ dimensional vector $\boldsymbol{r}$ of bead coordinates to that of dimensionless normal coordinates $\boldsymbol{U}$

$$
\mathbf{r}=b_{0} \mathbf{Q} \mathbf{u}
$$

and $\mathbf{Q}^{T}$ its transpose.

Equation 3 completely differs from the original estimate [3] that in first approximation the diagonal elements of the matrix $\mathbf{M}$ equal the eigenvalues $\lambda_{p R}$ of the Rouse model with vanishing hydrodynamic interaction, i.e.

$$
\mu_{p Z}^{(1)}=\lambda_{p R}=4 \sin ^{2}[p \pi / 2(Z+1)]
$$

and hence

$$
\nu_{p Z}^{(1)}=\lambda_{p Z} / \lambda_{p R}
$$




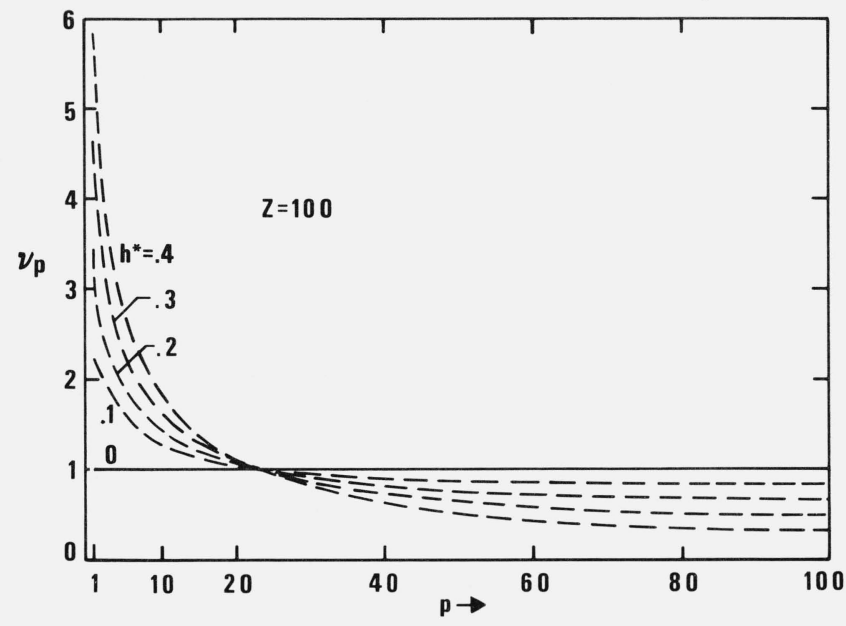

FIGURE 1. Diagonal elements $\nu_{\mathrm{p}}$ (new theory) and $\nu_{p}^{(1)}=\lambda_{\mathrm{pz}} / \lambda_{\mathrm{pR}}$ (old theory, broken lines) for $\mathrm{Z}=100$ and $\mathrm{h}^{*}=0, .1, .2, .3, .4$.

For $h^{*}=0$ (Rouse) the values of both theories coincide.

The subscripts $Z$ and $R$ refer to Zimm and Rouse [9] model, respectively. Such an estimate was based [3] on the supposition that the transformation matrix $\mathbf{Q}$ changes so little by the introduction of hydrodynamic interaction that $\mathbf{M}$ remains practically the same as in the free draining case, i.e. $\mathbf{M}=$ $\Lambda_{R}$.

The diagonal elements $\nu_{p}$ for $Z=100$ and $h^{*}=0$ (Rouse), $.1, .2, .3$, and $.4($ Zimm) are plotted in figure 1 and the eigenvalues $\lambda_{p}$ collected in table 1 . The old values of $\nu_{p}^{(1)}=$ $\lambda_{p Z} / \lambda_{p R}$ are connected with a broken line. They are partially situated below and partially above $\nu_{p}=1$. This reduces the differences between the old and new values in quantities dependent on $\nu_{p}$. They show up in the excess stress tensor as soon as internal viscosity, defined by the frictional parameter $\varphi$, is explicitly considered. The very large old values $\nu_{p}$ for small $p$ do not matter very much because in all formulae they are multiplied by very small values $\varphi_{p}=p \varphi / Z$. Since the differences between old and new $\nu_{p}$ increase with $h^{*}$, in that which follows, the comparison of calculated effects will be mainly done for $h^{*}=.4$, i.e., for a very large hydrodynamic interaction. The differences are smaller for smaller $h^{*}$ and of course disappear for the free draining coil with $h^{*}=0$ where $\lambda_{p Z}=\lambda_{p R}$.

The new values $\mu_{p}$ and $\nu_{p}$ do not enter the conventional expressions for the intrinsic stress or birefringence tensor of perfectly flexible necklace model so that no changes occur in these quantities. The situation is completely different if one considers the effects of internal viscosity which depend on $\nu_{p}$ [10-26]. They will be most conspicuous in the values of viscosity corresponding to high frequency flow field and in the phase angle between the stress and strain rate or between the birefringence and strain rate.

The interaction tensor $\mathbf{H}$ depends on the inverse intrabead distances $1 / r_{j k}$ which makes the hydrodynamic diffusion equation intrinsically non-linear. By replacing $1 / r_{j k}$ with its average value $\left\langle 1 / r_{j k}\right\rangle$ the tensor $\mathbf{H}$ becomes a constant which makes the diffusion equation linear and hence allows the introduction of normal coordinates according to eqs (1) to (4).
Such a procedure eliminates the possibility of any realistic consideration of the gradient dependence of any rheological or rheooptical effect because it does not take into account the change of shape of the random coil in flow which expands the molecule and hence increases the interbead distances $r_{j k}$. Note also that by preaveraging over all angles between the velocity and the interbead vector the formulation of $\mathbf{H}$ as function of $1 / r_{j k}$ completely evades the consideration of anisotropy of hydrodynamic interaction which by itself yields a gradient dependence of intrinsic viscosity [27] large enough for explaining experimental data.

The general toleration of such a profound modification of hydrodynamic interaction by the replacement of $1 / r_{j k}$ with its average $\left\langle 1 / r_{j k}\right\rangle$ makes hard to understand the almost general objections to the introduction of internal viscosity as a resistance of the necklace model against the deformational component of the normal eigenmodes [28-30]. If one accepts the rather questionable linearization of the hydrodynamic diffusion equation one has to accept also the next step, i.e., the concept of internal viscosity based on this linearity and its introduction in such a manner that the mathematical treatment remains as simple as possible.

In that which follows the results of the new theory will be compared with those of the old one for $h^{*}=.4$ and $Z=100$ in the whole frequency range and the dependence of the limiting values for $\omega=\infty$ on $Z$ in the range between $Z=3$ and 300 and on $h^{*}$ in the range between .1 and .4. In all cases the ratio between the internal viscosity coefficient $\varphi$ and the frictional coefficient of the bead $f=6 \pi a_{h} \eta_{s}$ will be assumed constant, $\varphi / f=2$. Here $\eta_{s}$ is viscosity of the solvent. The subscript $s$ applies to the properties of the solvent. The corresponding non-subscripted quantities relate to solution.

\section{Internal Viscosity}

The concept of internal viscosity was introduced in order to express the inability of the randomly coiled polymer molecule to change rapidly its shape $[31,32,10,11]$. Such changes occur during the rotation of the macromolecule in a flow with a rotational component, e.g., the laminar flow with transverse gradient, when the individual segments are alternatively passing from the direction of compression to the direction of extension and vice versa. The directions of maximum compression and extension of the volume element are in the flow plane perpendicular to each other. The rapidity of change is given by the transverse gradient which equals twice the angular velocity of the ideally flexible coil which rotates with the volume element. The other case is the oscillating flow field where the oscillation frequency determines the rapidity of change from compression to extension and vice versa.

In the limiting cases of zero gradient and zero frequency the deformation inability of the macromolecule does not play any role. All the changes occur so slowly that the effects are identical for completely rigid and ideally flexible coils if only their conformational distributions agree with each other. With increasing gradient and/or frequency, however, the time effects are playing a gradually increasing role. They are maximum in the second Newtonian regime corresponding to $\dot{\gamma}$ $\rightarrow \infty$ or $\omega \rightarrow \infty$.

The rigidity of the macromolecule can be assigned to different properties of the chain. It can be caused by the 
TABLE 1. Eigenvalues $\lambda_{p}$ for $Z=100$ and $h^{*}=0$ (Rouse), .1, .2,.3,.4 ( $\mathrm{Zimm}$ )

\begin{tabular}{|c|c|c|c|c|c|c|c|c|c|c|c|}
\hline$p$ & $H^{*}=0$ & .1 & .2 & .3 & .4 & $p$ & $h^{*}=0$ & .1 & .2 & .3 & .4 \\
\hline 1 & 0.000967 & 0.002170 & 0.003345 & 0.004506 & 0.005643 & 51 & 2.03111 & 1.7822 & 1.5334 & 1.2846 & 1.0359 \\
\hline 2 & .003869 & .007318 & .010718 & .014084 & .017417 & 52 & 2.09329 & 1.8320 & 1.5707 & 1.3094 & 1.0483 \\
\hline 3 & .008701 & .014650 & .020541 & .026383 & .032173 & 53 & 2.15538 & 1.8815 & 1.6077 & 1.3339 & 1.0602 \\
\hline 4 & .015460 & .023986 & .032447 & .040848 & .049177 & 54 & 2.21731 & 1.9308 & 1.6443 & 1.3579 & 1.0716 \\
\hline 5 & .024139 & .035175 & .046144 & .057043 & .067854 & 55 & 2.27904 & 1.9799 & 1.6807 & 1.3816 & 1.0826 \\
\hline 6 & .034730 & .048140 & .061482 & .074746 & .087907 & 56 & 2.34050 & 2.0286 & 1.7167 & 1.4049 & 1.0932 \\
\hline 7 & .047220 & .062802 & .078315 & .093745 & .10906 & 57 & 2.40153 & 2.0770 & 1.7523 & 1.4277 & 1.1033 \\
\hline 8 & .061602 & .079111 & .096552 & .11391 & .131113 & 58 & 2.46236 & 2.1249 & 1.7875 & 1.4502 & 1.1130 \\
\hline 9 & .077859 & .097010 & .11610 & .13509 & .15395 & 59 & 2.52265 & 2.1725 & 1.8223 & 1.4722 & 1.1223 \\
\hline 10 & .095974 & .11646 & .13688 & 15721 & .17740 & 60 & 2.58244 & 2.2195 & 1.8566 & 1.4938 & 1.1311 \\
\hline 11 & .115932 & .13742 & .15884 & .18017 & .20135 & 61 & 2.64166 & 2.2661 & 1.8905 & 1.5150 & 1.1396 \\
\hline 12 & .137713 & .15984 & .18192 & .20390 & .22571 & 62 & 2.70026 & 2.3120 & 1.9238 & 1.5357 & 1.1477 \\
\hline 13 & .161295 & .18370 & .20605 & .22831 & .25040 & 63 & 2.75819 & 2.3574 & 1.9566 & 1.5559 & 1.1554 \\
\hline 14 & .186656 & .20895 & .23120 & .25335 & .27533 & 64 & 2.81538 & 2.4021 & 1.9889 & 1.5757 & 1.1627 \\
\hline 15 & .213771 & .23556 & .25730 & .27896 & .30044 & 65 & 2.87178 & 2.4462 & 2.0206 & 1.5950 & 1.1697 \\
\hline 16 & .242614 & .26350 & .28434 & .30509 & .32566 & 66 & 2.92734 & 2.4895 & 2.0517 & 1.6139 & 1.1763 \\
\hline 17 & .273158 & .29272 & .31224 & .33169 & .35095 & 67 & 2.98200 & 2.5321 & 2.0821 & 1.6323 & 1.1826 \\
\hline 18 & .305372 & .32320 & .34099 & .35870 & .37623 & 68 & 3.03571 & 2.5738 & 2.1120 & 1.6502 & 1.1885 \\
\hline 19 & .339225 & .35490 & .37053 & .38610 & .40148 & 69 & 3.08842 & 2.6148 & 2.1411 & 1.6676 & 1.1941 \\
\hline 20 & .374681 & .38778 & .40084 & .41383 & .42664 & 70 & 3.14007 & 2.6548 & 2.1696 & 1.6845 & 1.1995 \\
\hline 21 & .411719 & .42180 & .43186 & .44186 & .45168 & 71 & 3.19063 & 2.6940 & 2.1974 & 1.7009 & 1.2045 \\
\hline 22 & .450288 & .45694 & .46358 & .47015 & .47655 & 72 & 3.24003 & 2.7323 & 2.2245 & 1.7168 & 1.2092 \\
\hline 23 & .490356 & .49316 & .49594 & .49867 & .50123 & 73 & 3.28823 & 2.7695 & 2.2508 & 1.7322 & 1.2137 \\
\hline 24 & .531886 & .53041 & .52892 & .52738 & .52568 & 74 & 3.33518 & 2.8058 & 2.2764 & 1.7471 & 1.2179 \\
\hline 25 & .574835 & .56867 & .56248 & .55626 & .54987 & 75 & 3.38085 & 2.8410 & 2.3012 & 1.7614 & 1.2218 \\
\hline 26 & .619163 & .60788 & .59659 & .58527 & .57378 & 76 & 3.42518 & 2.8752 & 2.3252 & 1.7753 & 1.2255 \\
\hline 27 & .664827 & .64802 & .63122 & .61438 & .59738 & 77 & 3.46812 & 2.9083 & 2.3484 & 1.7886 & 1.2290 \\
\hline 28 & .711782 & .68905 & .66632 & .64356 & .62065 & 78 & 3.50965 & 2.9402 & 2.3708 & 1.8014 & 1.2322 \\
\hline 29 & .759984 & .73093 & .70187 & .67279 & .64357 & 79 & 3.54972 & 2.9710 & 2.3924 & 1.8137 & 1.2352 \\
\hline 30 & .809386 & .77361 & .73784 & .70205 & .66613 & 80 & 3.58829 & 3.0007 & 2.4131 & 1.8255 & 1. 2380 \\
\hline 31 & .859939 & .81706 & .77419 & .73130 & .68829 & 81 & 3.62532 & 3.0291 & 2.4329 & 1.8367 & 1.2406 \\
\hline 32 & .911596 & .86124 & .81089 & .76053 & .71005 & 82 & 3.66078 & 3.0563 & 2.4518 & 1.8474 & 1.2430 \\
\hline 33 & .964305 & .90610 & .84790 & .78970 & .73140 & 83 & 3.69464 & 3.0823 & 2.4699 & 1.8575 & 1. 2453 \\
\hline 34 & 1.01802 & .95160 & .88521 & .81881 & .75231 & 84 & 3.72685 & 3.1069 & 2.4870 & 1.8672 & 1. 2474 \\
\hline 35 & 1.07268 & .99771 & .92277 & .84782 & .77279 & 85 & 3.75737 & 3.1303 & 2.5033 & 1.8762 & 1.2493 \\
\hline 36 & 1. 12824 & 1.0444 & .96055 & .87672 & .79281 & 86 & 3.78624 & 3.1524 & 2.5186 & 1.8848 & 1.2510 \\
\hline 37 & 1.18464 & 1.0916 & .99852 & .90548 & .81238 & 87 & 3.81335 & 3.1731 & 2.5329 & 1.8928 & 1.2526 \\
\hline 38 & 1.24183 & 1.1392 & 1.0367 & .93410 & .83148 & 88 & 3.83871 & 3.1925 & 2.5464 & 1.9002 & 1.2541 \\
\hline 39 & 1.29975 & 1.1873 & 1.0749 & .96254 & .85011 & 89 & 3.86229 & 3.2105 & 2.5588 & 1.9071 & 1.2554 \\
\hline 40 & 1.35835 & 1.2358 & 1.1133 & .99079 & .86826 & 90 & 3.88407 & 3.2272 & 2.5703 & 1.9134 & 1.2566 \\
\hline 41 & 1.41758 & 1.2846 & 1.1517 & 1.0188 & .88592 & 91 & 3.90403 & 3.2424 & 2.5808 & 1.9192 & 1.2577 \\
\hline 42 & 1.47736 & 1.3338 & 1.1902 & 1.0467 & .90311 & 92 & 3.92215 & 3.2563 & 2.5904 & 1.9245 & 1.2586 \\
\hline 43 & 1.53758 & 1.3832 & 1.2287 & 1.0742 & .91980 & 93 & 3.93840 & 3.2687 & 2.5989 & 1.9292 & 1.2595 \\
\hline 44 & 1.59839 & 1.4328 & 1.2671 & 1.1016 & .93601 & 94 & 3.95278 & 3.2796 & 2.6065 & 1.9333 & 1.2602 \\
\hline 45 & 1.65952 & 1.4825 & 1.3056 & 1.1286 & .95173 & 95 & 3.96527 & 3.2892 & 2.6130 & 1.9369 & 1.2608 \\
\hline 46 & 1.72098 & 1.5324 & 1.3439 & 1.1554 & .96696 & 96 & ק.97586 & 3.2972 & 2.6186 & 1.9400 & 1.2614 \\
\hline 47 & 1.78270 & 1.5824 & 1.3821 & 1.1819 & .98171 & 97 & 3.98454 & 3.3038 & 2.6232 & 1.9425 & 1.2618 \\
\hline 48 & 1.84464 & 1.6324 & 1.4202 & 1.2021 & .99597 & 98 & 3.99130 & 3.3090 & 2.6267 & 1.9444 & 1.2621 \\
\hline 49 & 1.90673 & 1.6824 & 1.4581 & 1.2339 & 1.0098 & 99 & 3.99613 & 3.3127 & 2.6292 & 1.9458 & 1.2624 \\
\hline 50 & 1.96890 & 1.7324 & 1.4959 & 1.2594 & 1.0231 & 100 & 3.99903 & 3.3149 & 2.6308 & 1.9466 & 1.2625 \\
\hline
\end{tabular}

energy barrier separating the gauche and trans conformations which makes any conformational change more time consuming than in the case of perfectly soft model [10]. Since the height of the energy barrier is independent of the viscosity of the solvent its relative effect as measured by the ratio of internal frictional resistance $\varphi$ caused by the barrier and the frictional resistance $f$ of the segment decreases with the viscosity of the solvent. The macromolecule acts as very rigid in a low viscosity liquid, e.g., acetone with $\eta_{s}=.322$ cPoise $=32.2 \mathrm{mNs} / \mathrm{m}^{3}$, and as very flexible in a high viscosity solvent, e.g., Aroclor with $\eta_{s}$ up to 100 Poise $\left(=1 \mathrm{kNs} / \mathrm{m}^{3}\right)$ and higher.
Another cause of slow molecular response to the rapidly changing flow field resides with the conformational restraints of the chain which permit only an interchange of gauche and trans conformations [20, 23, 24]. With almost rigid length of valency bonds this means that most changes of length and position of any chain segment require a much larger segment displacement than formulated in the ideally flexible necklace model which does not consider any inherent limitation of bead motion. Generally an axial displacement of the segment requires also some lateral displacement and vice versa. As a consequence the resistance of beads to position change is larger than assumed on the basis of hydrodynamic radius $a_{h}$. 
The ratio of the so obtained coefficient $\varphi$ to $f$ is independent of solvent viscosity because $\varphi$ and $f$ are both proportional to $\eta_{s}$. Their ratio just measures the ratio of true displacement to the minimum displacement explicitely considered in the diffusion equation. It seems to be close to 2 for vinyl polymers

The effect of internal viscosity is formulated in the system of normal modes [10]. For the $p$ th normal mode of deformation one has a resistance coefficient $\varphi p / Z$. Such a choice is reasonable for both origins of internal viscosity as just discussed. In the first case one can argue that the changes to comply with any deformational mode are linearly increasing with the number of chain atoms between subsequent nodes, i.e., with $Z / p$, because a conformational change takes place with equal probability at any of these chain atoms. This makes the resistance increase with $p / Z$. In the second case the displacement at lower modes can be achieved in many ways so that the actual lengthening of displacement path is much less noticeable than at higher modes where the conformational restrictions are soon becoming of overwhelming importance.

One may argue that the whole concept of internal viscosity can be discarded because it is not based on some strictly fundamental analysis of chain dynamics. It was indeed introduced in a rather pragmatic manner which also permitted an easy mathematical treatment [10]. But it turns out that all more detailed treatments of Brownian motion of beads or of correlation between the motion of two or more beads [33-35] lead to some, often hidden, statement of molecular rigidity which is needed for the results of such a study to reproduce the characteristic rheological features of polymer systems, e.g., the non-vanishing limiting intrinsic viscosity at very high frequency [36-51]. Such a state of affair seems more to support than to refute the concept of internal viscosity in spite of its more pragmatic than fundamental way of introduction.

\section{The Distribution Function of the Beads}

The continuity equation of the ideally flexible necklace in laminar flow which determines the distribution function $\psi\left(\mathbf{r}_{0}\right.$, $\boldsymbol{r}_{1} \cdots \boldsymbol{r}_{z}$ ) reads

$$
\begin{aligned}
& \boldsymbol{\nabla}_{r}^{T}\left[\mathbf{v} \psi-\left(3 D_{0} / b_{0}^{2}\right) \mathbf{H A} \boldsymbol{r} \psi-D_{0} \mathbf{H} \boldsymbol{\nabla}_{r} \psi\right] \\
&=-\partial \psi / \partial t .
\end{aligned}
$$

Here $D_{0}=k T / f$ is the translational diffusion coefficient of the bead. By introduction of normal coordinates, eq (4), one transforms eq (7) into a system of $Z+1$ partial differential equations

$$
\begin{aligned}
\boldsymbol{\nabla}_{p}\left[\mathbf{v}_{p} \psi_{p}-\left(3 D_{0} / b_{0}^{2}\right) \lambda_{p} \mathbf{u}_{p}-\left(D_{0} / b_{0}{ }^{2}\right) \nu_{p}\right. & \left.\nabla_{p} \psi_{p}\right] \\
& =-\partial \psi_{p} / \partial t
\end{aligned}
$$

each depending only on the coordinates of the $p$ th eigenmode. Note that $\mathbf{v}_{p}$ in normal coordinates has the dimension $s^{-1}$. The distribution function of the coil is the product of all $\psi_{p}$

$$
\psi(\boldsymbol{u}, t)=\psi_{1}\left(\boldsymbol{u}_{l}, t\right) \cdots \psi_{Z}\left(\boldsymbol{u}_{Z}, t\right)
$$

The functions $\psi_{p}$ depend on the kind of flow field $\mathbf{v}$. The 0 th mode does not show up in $\psi$ because it represents a uniform translation of the whole necklace which does not affect $\psi$.
The introduction of internal viscosity adds a viscous type resistance coefficient $\varphi_{p}=p \varphi / Z$ opposing the $p$ th eigenmode of the true deformation rate of the coil. This rate is obtained by subraction of pure rotational velocity $\Omega \times \mathbf{u}_{p}$ from the total deformation rate $\partial \boldsymbol{u}_{p} / \partial t$. This yields an internal viscosity force $[10,11]$

$$
\boldsymbol{F}_{i p}=-(p \varphi / Z)\left(\partial \boldsymbol{u}_{p} / \partial t-\boldsymbol{\Omega} \times \boldsymbol{u}_{p}\right) b_{0}
$$

If one introduces this force in the $p$ th diffusion equation (eq (8)) one obtains after some rearrangements

$$
\begin{aligned}
\left(1+\nu_{p} \varphi_{p} / f\right)^{-1} & \nabla_{p}\left\{\left[\mathbf{v}_{p}-\left(3 D_{0} / b_{0}^{2}\right) \lambda_{p} \boldsymbol{u}_{p}+\left(\nu_{p} \varphi_{p} / f\right) \Omega\right.\right. \\
& \left.\left.\times \mathbf{u}_{p}-\left(D_{0} / b_{0}^{2}\right) \nu_{p} \boldsymbol{\nabla}_{p}\right] \psi_{p}\right\}=-\partial \psi / \partial t
\end{aligned}
$$

The distribution function $\psi_{p}$ depends on the kind of flow and on the angular velocity vertor $\Omega$.

In a jet or plane flow with longitudinal gradient without a rotational flow component one has $\Omega=0$. In a flow with transverse gradient, $\mathbf{v}=\dot{\gamma}(\mathbf{y}, \mathrm{o}, \mathrm{o})$, the angular velocity equals $-\gamma / 2$

$$
\boldsymbol{\Omega}=(0,0,-\dot{\gamma} / 2)
$$

for relatively soft molecules which rotate in phase with the volume element. This is the case with practically all conventional macromolecules if the degree of polymerization is so high that a truly random coil is formed. Very short chains, chains with a great many double bonds, ladder type and multiple strand molecules, however, are more rigid and tend to rotate with a non-uniform angular velocity which depends on orientation of the molecule. It is different from that of he volume element. Assymptotically, at very high rigidity and fully extended shape of the macromolecule, it approaches that of rigid bodies, e.g., rods or ellipsoides. In that which follows only the case of practically undeformed relatively soft coils with $\Omega=-\dot{\gamma} / 2$ will be considered.

In the steady state flow with transverse gradient the $p$ th eigenmode distribution function of the soft necklace reads

$$
\begin{aligned}
& \psi_{p}\left(\xi_{p}, \eta_{p}, \xi_{p}\right)=\left(\frac{\mu_{p}}{\pi}\right)^{3 / 2}\left(\frac{1+\beta_{p}^{\prime 2}-\beta_{p}^{2}}{1+\beta_{p}^{\prime 2}}\right)^{1 / 2} \\
& \exp \left(-\frac{\mu_{p}}{1+\beta_{p}^{\prime 2}}\left\{\left(1+\beta_{p}^{\prime 2}-\beta_{p}^{2}\right) \xi_{p}{ }^{2}-2 \beta_{p} \xi_{p} \eta_{p}\right.\right. \\
&\left.\left.\left.+\left[1+\beta_{p}^{\prime}\left(\beta_{p}^{\prime}+\beta_{p}\right)\right] \eta_{p}^{2}\right)+\left(1+\beta_{p}^{\prime 2}\right) \zeta_{p}{ }^{2}\right\}\right) \\
& \beta_{p}=\dot{\gamma} b_{0}^{2} / 6 D_{0} \lambda_{p} \\
& \beta_{p}^{\prime}=\beta_{p}\left(1+\nu_{p} \varphi_{p} / f\right) .
\end{aligned}
$$

The index $p$ runs from 1 to $Z$. The value 0 is excluded. Without internal viscosity, $\varphi_{p}=0$, one has $\beta^{\prime}{ }_{p}=\beta_{p}$ and one obtains the conventional distribution function. Note that eq (13) and the distribution function eq (6.1) in Ref. [1] refer to different flow fields: $\dot{\gamma}(y, o, o)$ in the former and $\gamma(z, o, o)$ in the latter case. 
Equation (13) explicitely contains the diagonal terms $\lambda_{p}$, $\mu_{p}$ and $\nu_{p}$. In the averages (dyadic formulation)

$$
\left\langle\boldsymbol{r}^{\mathrm{T}} \mathbf{A} \boldsymbol{r}\right\rangle=b_{0}{ }^{2}\left\langle\boldsymbol{u}^{\mathrm{T}} \mathbf{M} \boldsymbol{u}\right\rangle=b_{0}{ }^{2} \sum_{\boldsymbol{p}=1}^{Z} \mu_{p}\left\langle\boldsymbol{u}_{p} \boldsymbol{u}_{p}\right\rangle
$$

which appear in the intrinsic stress and optical tensor the coefficients $\mu_{p}$ drop completely because the averages $\left\langle\boldsymbol{u}_{p} \boldsymbol{U}_{p}\right\rangle$ are proportional to $1 / \mu_{p}$. The coefficients $\nu_{p}$, however, remain in $\beta^{\prime}{ }_{p}$ as long as $\varphi \neq 0$. As already mentioned they are equal to unity (new theory) while formerly they were approximated by $\left(\lambda_{Z} / \lambda_{R}\right)_{p}=\lambda_{p}\left(h^{*}\right) / \lambda_{p}\left(h^{*}=0\right) \neq 1$ (old theory).

In the oscillating flow field the gradient is a function of time

$$
\dot{\gamma}=\gamma_{0} \mathrm{e}^{i \omega t}
$$

The amplitude $\dot{\gamma}_{0}$ is so small that the molecule remains practically undeformed so that the zero gradient eigenvalues $\lambda_{p}$ and diagonal elements $\nu_{p}$, calculated for the coil at rest, are applicable.

\section{The Intrinsic Stress Tensor}

In dyadic formulation the intrinsic stress tensor reads

$$
\begin{aligned}
{[\boldsymbol{\sigma}]=\lim _{c \rightarrow 0} \frac{\boldsymbol{\sigma}-\boldsymbol{\sigma}_{s}}{c}=\frac{N}{M}\left\langle\boldsymbol{r}^{\mathrm{T}} \boldsymbol{F} .\right.} & \begin{array}{c}
\text { In the case of laminar flow with } \\
\dot{\gamma}(\mathbf{y}, 0,0), \text { one obtains the set of linear }
\end{array} \\
\left\langle\xi_{p}{ }^{2}\right\rangle-\nu_{p} / 3 \lambda_{p}-2 \tau_{p} \dot{\gamma}\left(1+\nu_{p} \varphi_{p} / 2 f\right)\left\langle\xi_{p} \eta_{p}\right\rangle & =-\tau_{p}^{\prime} d\left\langle\xi_{p}{ }^{2}\right\rangle / d t
\end{aligned} \mid \begin{aligned}
\left\langle\xi_{p} \eta_{p}\right\rangle-\tau_{p} \gamma\left(\nu_{p} \varphi_{p} / 2 f\right)\left\langle\xi_{p}{ }^{2}\right\rangle-\tau_{p} \dot{\gamma}\left(1+\nu_{p} \varphi_{p} / 2 f\right)\left\langle\eta_{p}{ }^{2}\right\rangle & =-\tau_{p}^{\prime} d\left\langle\xi_{p} \eta_{p}\right\rangle / d t \\
\left\langle\eta_{p}{ }^{2}\right\rangle-\nu_{p} / 3 \lambda_{p}+\tau_{p} \dot{\gamma}\left(\nu_{p} \varphi_{p} / f\right)\left\langle\xi_{p} \eta_{p}\right\rangle & =-\tau_{p}^{\prime} d\left\langle\eta_{p}{ }^{2}\right\rangle / d t \\
\left\langle\eta_{p} \zeta_{p}\right\rangle+\tau_{p} \dot{\gamma}\left(\nu_{p} \varphi_{p} / 2 f\right)\left\langle\zeta_{p} \xi_{p}\right\rangle & =-\tau_{p}^{\prime} d\left\langle\eta_{p} \zeta_{p}\right\rangle / d t \\
\left\langle\zeta_{p}{ }^{2}\right\rangle-\nu_{p} / 3 \lambda_{p} & =-\tau_{p}^{\prime} d\left\langle\zeta_{p}{ }^{2}\right\rangle / d t \\
\left\langle\zeta_{p} \xi_{p}\right\rangle+\tau_{p} \dot{\gamma}\left(1+\nu_{p} \varphi_{p} / 2 f\right)\left\langle\eta_{p} \zeta_{p}\right\rangle & =-\tau_{p}^{\prime} d\left\langle\zeta_{p} \xi_{p}\right\rangle / d t
\end{aligned}
$$

The steady state solutions which apply after the complete

decay of transient phenomena read

$$
\begin{aligned}
\left\langle\xi_{p}{ }^{2}\right\rangle & =\frac{1}{3 \mu_{p}}\left[1+\frac{\gamma_{0}^{2} \tau_{p}\left(\tau_{p}^{\prime}+\tau_{p}\right) / 2}{1+i \omega \tau_{p}^{\prime}}\left(1+\frac{e^{2 i \omega t}}{1+2 i \omega \tau_{p}^{\prime}}\right)\right] \\
\left\langle\xi_{p} \eta_{p}\right\rangle & =\frac{1}{3 \mu_{p}} \frac{\gamma_{0} \tau_{p}}{1+i \omega \tau_{p}^{\prime}} e^{i \omega t} \\
\left\langle\eta_{p}^{2}\right\rangle & =\frac{1}{3 \mu_{p}}\left[1-\frac{\gamma_{0}^{2} \tau_{p}\left(\tau_{p}^{\prime}-\tau_{p}\right) / 2}{1+i \omega \tau_{p}^{\prime}}\left(1+\frac{e^{2 i \omega t}}{1+2 i \omega \tau_{p}^{\prime}}\right)\right] \\
\left\langle\zeta_{p}{ }^{2}\right\rangle & =\frac{1}{3 \mu_{p}} \\
\left\langle\eta_{p} \zeta_{p}\right\rangle & =\left\langle\zeta_{p} \xi_{p}\right\rangle=0
\end{aligned}
$$


The averages are cut off beyond the lowest power $\dot{\gamma}$ which is needed later in zero gradient expressions for intrinsic viscosity, normal stress difference and birefringence. These averages have to be inserted in the expression for the intrinsic stress tensor.

$$
\begin{aligned}
& {[\sigma]=(N / M)\left[3 k T\left\langle\mathbf{u}^{\mathrm{T}} \mathbf{M} \mathbf{u}\right\rangle\right.} \\
& \left.\quad+\left(\dot{\gamma} b_{0}{ }^{2} / 2\right)\left\langle\mathbf{u}^{\mathrm{T}} \boldsymbol{\phi}(\boldsymbol{\eta}, \boldsymbol{\xi}, o)\right\rangle-k T \mathbf{1}\right] \times(\mathbf{1}+\mathbf{N} \boldsymbol{\Phi} / f)^{-1}
\end{aligned}
$$

yielding the frequency dependence of intrinsic viscosity

$$
\begin{aligned}
{[\eta] } & =[\eta]^{\prime}{ }_{\omega}-i[\eta]^{\prime \prime}{ }_{\omega} \\
& =\lim _{c \rightarrow 0} \frac{\eta-\eta_{s}}{c \eta_{s}}=\lim _{c \rightarrow 0} \frac{\sigma_{12}{ }^{*}-\sigma_{12 s}}{c \sigma_{12 s}}=\left[\sigma_{12}\right]^{*} / \eta_{s} \dot{\gamma} \\
& =\frac{R T}{M \eta_{s}} \sum_{p=1}^{Z}\left(\frac{3 \mu_{p}}{\dot{\gamma}}\left\langle\xi_{p} \eta_{p}\right\rangle+\frac{b_{0}{ }^{2} \varphi_{p}}{2 k T}\left\langle\eta_{p}{ }^{2}\right\rangle\right) /\left(1+\frac{\nu_{p} \varphi_{p}}{f}\right) \\
& =\frac{R T}{M \eta_{s}} \sum \tau_{p} \frac{1+i \omega\left(\tau_{p}^{\prime}-\tau_{p}\right)}{1+i \omega \tau_{p}^{\prime}} \\
& =\frac{R T}{M \eta_{s}}[\mathrm{I}+\mathrm{IV}-i(\mathrm{II}-\mathrm{III})] / \omega
\end{aligned}
$$

$\tan \delta_{\eta}=(\mathrm{II}-\mathrm{III}) /(\mathrm{I}+\mathrm{IV})$.

The absolute value of intrinsic viscosity $[\eta]_{\omega} M \eta_{s} / R T$ and the phase angle $\delta_{\eta}$ as function of $\omega \tau_{1}$ are plotted in figure 2 for $Z$ $=100, \varphi / f=2, h^{*}=.4$ according to old and new theory.

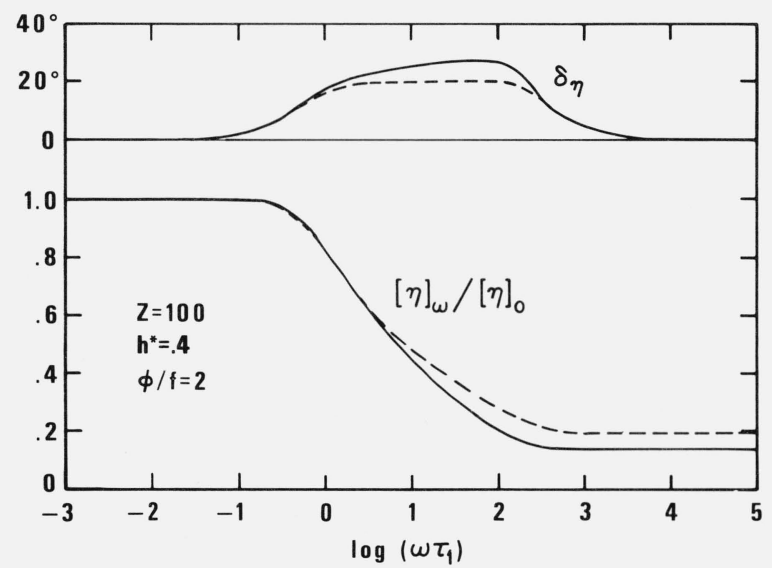

FIGURE 2. Relative intrinsic viscosity $[\eta]_{\omega} /[\eta]_{0}$ and phase angle $\delta_{\eta}$ for $\mathrm{Z}=100, \mathrm{~h}^{*}=.4$, and $\varphi / \mathrm{f}=2$ as function of $\omega \tau_{1}$ according to new (full line) and old (broken line) theory.
A rather similar but not identical expression applies to intrinsic streaming birefringence

$$
\begin{aligned}
{[\Delta n]_{\omega}^{*} } & =[\Delta n]_{\omega}^{\prime}-[\Delta n]_{\omega}^{\prime \prime} \\
& =\lim _{c \rightarrow 0} \frac{\Delta \mathrm{n}^{*}-\Delta \mathrm{n}_{s}}{\mathrm{cn}_{s} \eta_{s} \gamma}=\lim _{c \rightarrow 0} \frac{\Delta \mathrm{n}^{*}-\Delta \mathrm{n}_{s}}{\mathrm{cn}_{s} \sigma_{12 s}} \\
& =K \frac{R T}{M \eta_{s}} \sum_{p=1}^{Z} \frac{2 \mu_{p}}{\dot{\gamma}}\left\langle\xi_{p} \eta_{p}\right\rangle /\left(1+\frac{\nu_{p} \varphi_{p}}{f}\right) \\
& =\frac{4 \pi}{5}\left(\frac{n^{2}+2}{3 n}\right)^{2} \frac{\left.N \alpha_{1}-\alpha_{2}\right)}{M \eta_{s}} \sum \frac{\tau_{p}}{1+i \omega \tau_{p}^{\prime}} \\
& =K \frac{R T}{M \eta_{s}}(\mathrm{I}-i \mathrm{II}) / \omega
\end{aligned}
$$

$\tan \delta_{n}=\mathrm{II} / \mathrm{I}$.

In the case of dynamic birefringence $\Delta n$ is the difference between the refractive indices in the diagonal direction in the first and second quadrant. The extinction angle $\chi=45^{\circ}$ as long as the gradient amplitude $\dot{\gamma}$ is small enough. The birefringence $[\Delta n]_{\omega} M \eta_{s} / K R T$ and the phase angle $\delta_{n}$ are plotted in figure 3 for the same values of $Z, \varphi / f$ and $h^{*}$ as in figure 2 .

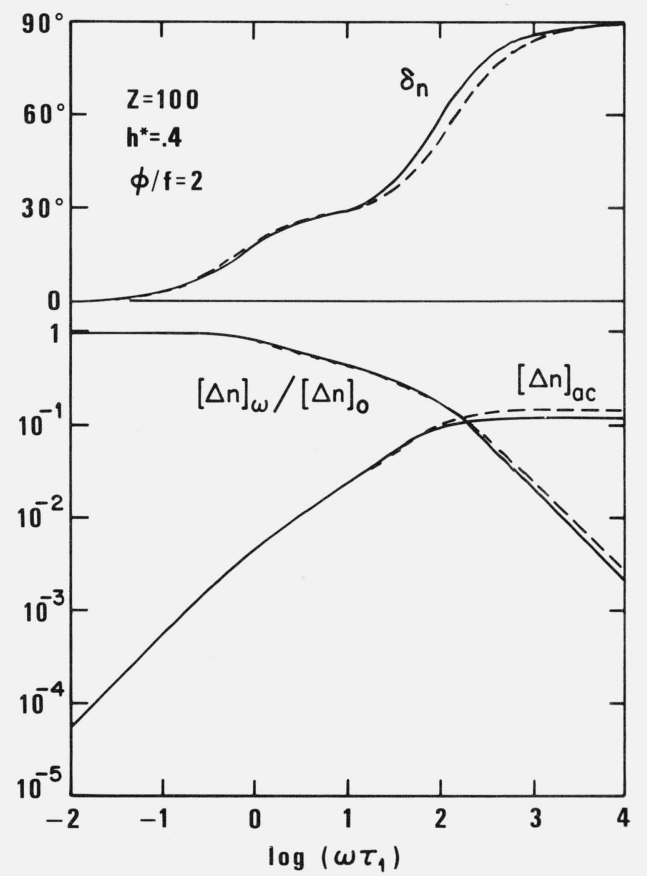

FigURE 3. Relative intrinsic streaming birefringence $[\Delta \mathrm{n}]_{\omega} /[\Delta \mathrm{n}]_{0}$ intrinsic accoustic birefringence $[\Delta n]_{\text {ac }}$ and phase angle $\delta_{\mathrm{n}}$ for $\mathrm{Z}=100, \mathrm{~h}^{*}=.4$, and $\varphi / \mathrm{f}=2$ as function of $\omega \tau_{1}$ according to new (full line) and old (broken line) theory. 
The meaning of the symbols in eqs (17) to (21) is as follows:

$$
\begin{aligned}
\tau_{p} & =b_{0}^{2} / 6 D_{0} \lambda_{p} \\
\tau_{p}^{\prime} & =\tau_{p}\left(1+\nu_{p} \varphi_{p} / f\right) \\
\tau_{p}^{\prime}-\tau_{p} & =\tau_{p} \nu_{p} \varphi_{p} / f \\
K & =\frac{4 \pi}{5}\left(\frac{n^{2}+2}{3 n}\right)^{2}\left(\frac{\alpha_{1}-\alpha_{2}}{k T}\right) \\
\mathrm{I} & =\sum \omega \tau_{p} /\left(1+\omega^{2} \tau_{p}^{\prime 2}\right) \\
\mathrm{II} & =\sum \omega^{2} \tau_{p} \tau_{p}^{\prime} /\left(1+\omega^{2} \tau_{p}^{\prime 2}\right) \\
\mathrm{III} & =\sum \omega^{2} \tau_{p}\left(\tau_{p}^{\prime}-\tau_{p}\right) /\left(1+\omega^{2} \tau_{p}^{\prime 2}\right) \\
\mathrm{IV} & =\sum \omega^{3} \tau_{p} \tau_{p}^{\prime}\left(\tau_{p}^{\prime}-\tau_{p}\right) /\left(1+\omega^{2} \tau_{p}^{\prime 2}\right)
\end{aligned}
$$

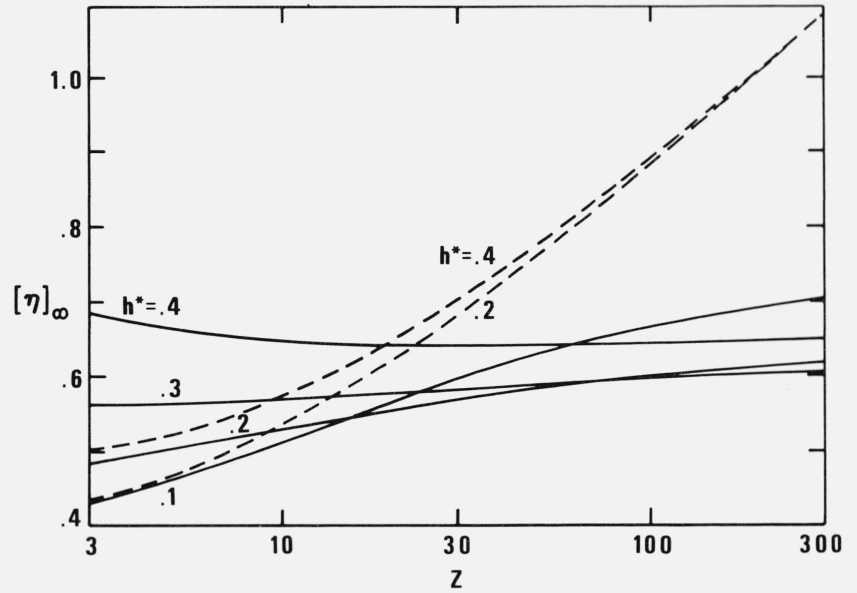

Figure 4. Second Newtonian intrinsic viscosity $[\eta]_{\infty}$ as function of number of links $\mathrm{Z}$ and hydrodynamic interaction $\mathrm{h}$ as parameter for $\varphi / \mathrm{f}=2$.

New (full line) and old (broken line) theory.
The sums III and IV go to zero for vanishing internal viscosity $\varphi \rightarrow 0$. In this limiting case the intrinsic viscosity and streaming birefringence are proportional to each other. Here $R$ is the gas constant, $K$ is the rheooptical coefficient, $n$ is refractive index, $\delta$ is the phase angle between the flow gradient and viscosity or birefringence, $\alpha_{1}$ and $\alpha_{2}$ are the optical polarizabilities of the link in the directions parallel and perpendicular to the link respectively.

Both definitions of intrinsic viscosity and birefringence in eqs (20) and (21) tend to make the intrinsic values as much as possible independent of the specific properties of the solvent, i.e., of $\eta_{s}$ and $n_{s}$ which influence the orientational forces, rigidity and optical anistropy of the dissolved macromolecule [52].

It is important to note that as a consequence of the new terms III and IV, i.e., $\left\langle\eta_{p}{ }^{2}\right\rangle$, caused by the introduction of internal viscosity the expressions for intrinsic viscosity and streaming birefringence, eq. (20) and (21), respectively, are not proportional to each other. Hence the rheooptical law does not apply to such a model. In particular $[\Delta n]_{\omega}$ cannot be written as $K[\eta] \omega \sim[\sigma]_{12}$.

The proportionality, however, still holds in the first Newtonian region, $\omega \rightarrow 0$, where all the terms but $I / \omega$ go to zero. This means that the intrinsic viscosity $[\eta]$ and the Maxwell constant $[\Delta n]$ and hence the stress and the optical tensor are proportional to each other for $\dot{\gamma} \rightarrow 0$ and $\omega \rightarrow 0$. This is the case in most applications of flow stress mapping by means of streaming birefringence of dilute polymer solutions.

With increasing frequency and finite internal viscosity, $[\Delta n]_{\omega}$ goes to zero while $[\eta]_{\omega}$ tends to finite value. The rheooptical law breaks down completely. In this second Newtonian range the intrinsic viscosity is independent of frequency. One derives from eq (20)

$$
[\eta]_{\infty}=\frac{R T}{M \eta_{s}} \sum_{p=1}^{Z} \frac{\tau_{p}}{1+f / \nu_{p} \varphi_{p}}
$$

The limiting values $[\eta]_{\infty} \mathrm{M} \eta_{s} / \mathrm{RT}$ for $Z$ between 3 and 300 and $h^{*}=.1, .2, .3, .4$ are plotted in figure 4 for the new (full line) and old (broken line) theory. One sees that the absolute values for $\nu_{p}=1$ and $\nu_{p}^{(1)}=\lambda_{p Z} / \lambda_{p R}$ differ by less than a factor of 2. But their dependence on $Z$, i.e., on molecular weight, is just the opposite for $h^{*}=.4$. In the incorrect formulation of the old theory $[\eta]_{\infty}$ slightly increases with $Z$ while in the correct formulation of the new theory it decreases with $Z$. Such a dependence on $Z$ is much more in agreement with experimental data on polystyrene in Aroclor [44]. The second Newtonian viscosity $[\eta]_{\infty}$ is $14.3 \mathrm{~cm}^{3} / \mathrm{g}$ if one goes with $M$ from 20,000 to 860,000 . The old theory yields a steady increase of $[\eta]_{\infty}$ with $M$ in sharp disagreement with these data.

The often used real and imaginary part of intrinsic shear modulus

$$
\begin{aligned}
{[G]^{*}=[G]^{\prime}+i[G]^{\prime \prime}=} & i \omega \eta_{s}[\eta]_{\omega}^{*} \\
& =\frac{1}{\dot{\gamma}}\left[\sigma_{12}\right]^{*}=\lim _{c \rightarrow 0} \frac{\sigma_{12}^{*}-\sigma_{12 s}}{c \dot{\gamma}}
\end{aligned}
$$

are plotted in figure 5. The difference between the old and new theory is relatively small for $Z=100$ but would be larger for $Z=300$.

The consequence of the non-vanishing second Newtonian viscosity, $[\eta]_{\infty} \neq 0$, is the linear increase of $[G]^{\prime}$ with $\omega \tau_{1}$ in the assymptotic high frequency region. Such a behavior is in perfect agreement with experimental data on polystyrene in highly viscous Aroclor [44-51]. These data together with the zero gradient intrinsic orientation data of streaming birefringence of polystyrene in solvents of increasing viscosity [53] constitute the main support of the theory. 


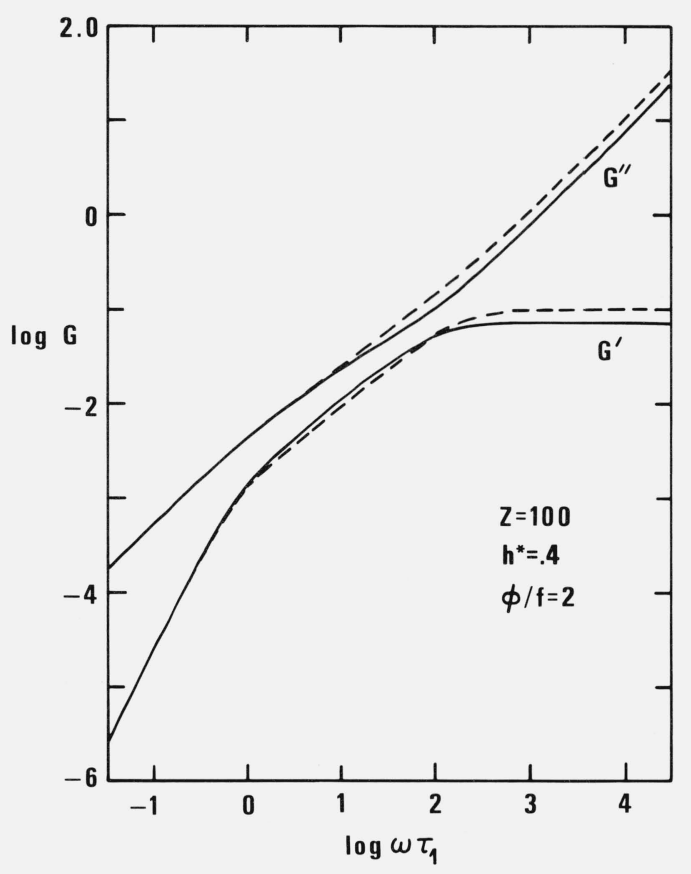

FIGURE 5. Intrinsic shear moduli $[\mathrm{G}]^{\prime}$ and $[\mathrm{G}]^{\prime \prime}$ for $\mathrm{Z}=$ $100, \mathrm{~h}^{*}=.4$, and $\varphi / \mathrm{f}=2$ as functions of $\omega \tau_{1}$ according to new (full line) and old (broken line) theory.

The intrinsic first normal stress difference turns out to be

$$
\begin{aligned}
{\left[\sigma_{11}-\sigma_{22}\right]^{*} } & \\
= & \frac{R T}{M} \sum_{p=1}^{Z}\left(3 \mu_{p}\left\langle\xi_{p}{ }^{2}-\eta_{p}{ }^{2}\right\rangle+\frac{\dot{\gamma} b_{0}{ }^{2} \varphi_{p}}{k T}\left\langle\xi_{p} \eta_{p}\right\rangle\right) / \\
& \left(1+\nu_{p} \varphi_{p} / f\right) \\
= & \frac{R T}{M} \gamma_{0}{ }^{2} \sum_{p=1}^{Z} \frac{\tau_{p}{ }^{2}}{1+i \omega \tau_{p}^{\prime}} \\
& \cdot\left[1+\left(\frac{1}{1+2 i \omega \tau_{p}^{\prime}}+\frac{\tau_{p}^{\prime}-\tau_{p}}{\tau_{p}}\right) e^{2 i \omega t}\right] \\
= & A^{*} e^{2 i \omega t}+B^{*}
\end{aligned}
$$

while the second normal stress difference vanishes in all isolated necklace models with ideal elastic links independent of internal viscosity. In applying eq (25) one must not forget that the phase angle $\delta_{1}$ of $A^{*}$ is dependent only on the factor of $\exp (2 i \omega t)$ and that the angle $\delta_{2}$ only reduces the constant vertical displacement to $B \cos \delta_{2}$ but does not yield any phase shift.

The term 1 in the parenthesis, i.e., $B^{*}$, keeps the first normal stress difference positive during the whole period up to very high frequencies. The oscillation is taking place with twice the frequency of the flow field. All these effects are very much the same as in the case of no internal viscosity. The difference is mainly in the replacement of $\tau_{p}$ by $\tau^{\prime}{ }_{p}$ and the term proportional to $\tau_{p}^{\prime}-\tau_{p}$. The amplitude $A$ of the oscillating term

$$
\begin{aligned}
A^{*}= & A e^{-i \delta_{1}} \\
= & \frac{R T}{M} \dot{\gamma}_{p}{ }^{2} \sum_{p=1}^{Z} \frac{\tau_{p}}{1+i \omega \tau_{p}^{\prime}}\left(\frac{1}{1+2 i \omega \tau_{p}^{\prime}}+\frac{\tau_{p}^{\prime}-\tau_{p}}{\tau_{p}}\right) \\
& {\left[1+2 \omega^{2} \tau_{p}^{\prime}\left(2 \tau_{p}^{\prime}-\tau_{p}\right)\right] } \\
= & \frac{R T}{M} \dot{\gamma}_{0}{ }^{2} \sum_{p=1}^{Z} \tau_{p}{ }^{2} \frac{-i \omega\left[\tau_{p}^{\prime}+2 \tau_{p}+4 \omega^{2} \tau_{p}^{\prime}{ }^{2}\left(\tau_{p}^{\prime}-\tau_{p}\right)\right]}{\left(1+\omega^{2} \tau_{p}^{\prime}{ }^{2}\right)\left(1+4 \omega^{2} \tau_{p}^{\prime}{ }^{2}\right)}
\end{aligned}
$$

is proportional to the square of the amplitude of the oscillating gradient as in the case of no internal viscosity. The value $\left(A / \gamma_{0}^{2}\right)(M / R T)$, the phase angle $\delta_{1}$, and the frequency independent displacement

$$
\left(B \cdot \cos \delta_{2} / \dot{\gamma}_{0}^{2}\right)(M / R T)=\sum_{p=1}^{Z} \frac{\tau_{p}^{2}}{1+\omega^{2} \tau_{p}^{\prime}{ }^{2}}
$$

are plotted in figure 6 as functions of $\omega \tau_{1}$ for $Z=100$ and $h^{*}$ $=.4$. Both quantities $A$ and $B$ are going to zero with increasing frequency. In contrast to intrinsic viscosity the limiting first normal stress difference, at $\omega \rightarrow \infty$, does not become finite by introduction of internal viscosity although it goes to zero more slowly, as $\omega^{-1}$ instead of as $\omega^{-3}$.

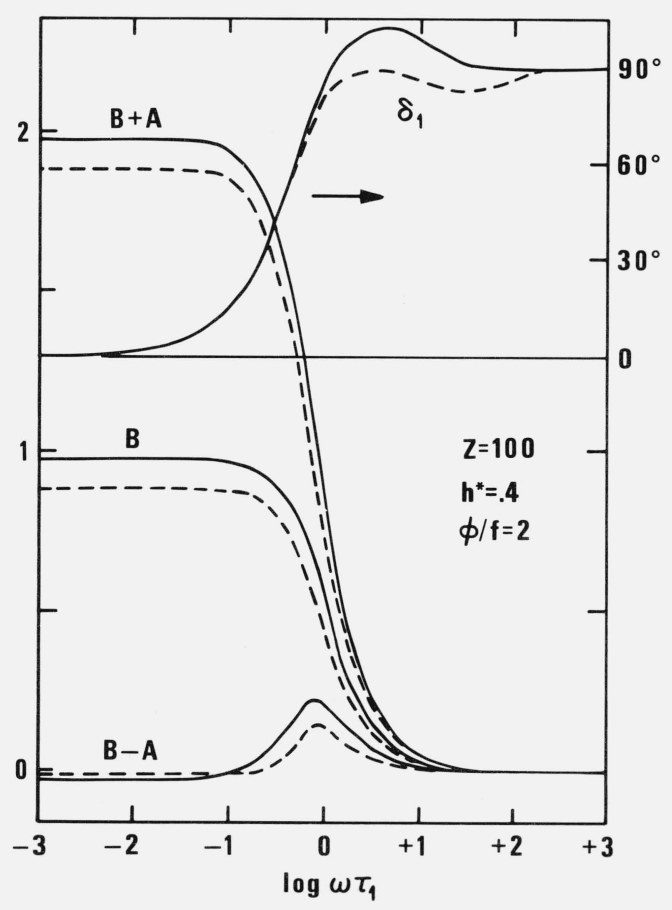

FigurE 6. The coefficient $\mathrm{B}$, the sum $\mathrm{B}+\mathrm{A}$, the difference $\mathrm{B}-\mathrm{A}$ of the relative intrinsic normal stress difference $\left[\sigma_{11}-\sigma_{22}\right]_{\omega} /\left[\sigma_{11}-\sigma_{22}\right]_{0}=\mathrm{A} \mathrm{e}_{1}{ }^{2 \mathrm{i} \omega \tau-\mathrm{i} \delta 1}+\mathrm{B}$ for $\mathrm{Z}=100, \mathrm{~h}^{*}$ $=.4$ and $\varphi / \mathrm{f}=2$ as functions of $\omega \tau_{1}$ according to new (full line) and old (broken line) theory. 


\section{Flow With Longitudinal Gradient}

In jet flow, $\mathbf{v}=\dot{\gamma}(-x / 2,-y / 2, z)$ the intrinsic stress tensor

$$
\begin{aligned}
& {[\boldsymbol{\sigma}]=\frac{N_{A}}{M}[3 k T\langle\mathbf{u} \mathbf{M} \mathbf{u}\rangle} \\
&+\left(\dot{\gamma} b_{0}{ }^{2} / 2\right)\langle\mathbf{u} \phi(-\boldsymbol{\xi},-\boldsymbol{\eta},+2 \boldsymbol{\eta})\rangle+k T \mathbf{1}] \\
& \times(\mathbf{1}+\mathbf{N} \boldsymbol{\phi} / f)^{-1}
\end{aligned}
$$

is independent of coil rotation in flow because the flow field has no rotational component, $\Omega=0$. The bilinear coordinate averages are derivable from the set of differential equations

$$
\begin{aligned}
& \left(1+\dot{\gamma} \tau_{p}\right)\left\langle\xi_{p}{ }^{2}\right\rangle-\nu_{p} / 3 \lambda_{p}=-\tau_{p}^{\prime} d\left\langle\xi_{p}{ }^{2}\right\rangle / d t \\
& \left(1+\gamma \tau_{p}\right)\left\langle\xi_{p} \eta_{p}\right\rangle=-\tau_{p}^{\prime} d\left\langle\xi_{p} \eta_{p}\right\rangle / d t \\
& \left(1+\dot{\gamma} \tau_{p}\right)\left\langle\eta_{p}{ }^{2}\right\rangle-\nu_{p} / 3 \lambda_{p}=-\tau_{p}^{\prime} d\left\langle\eta_{p}{ }^{2}\right\rangle / d t \\
& \left(1-\gamma \tau_{p} / 2\right)\left\langle\eta_{p} \zeta_{p}\right\rangle=-\tau_{p}^{\prime} d\left\langle\eta_{p} \zeta_{p}\right\rangle / d t \\
& \left(1-\dot{\gamma} \tau_{p}\right)\left\langle\zeta_{p}{ }^{2}\right\rangle-\nu_{p} / 3 \lambda_{p}=-\tau_{p}^{\prime} d\left\langle\zeta_{p}{ }^{2}\right\rangle / d t \\
& \left(1-\gamma \tau_{p} / 2\right)\left\langle\zeta_{p} \xi_{p}\right\rangle=-\tau_{p}^{\prime} d\left\langle\zeta_{p} \xi_{p}\right\rangle / d t
\end{aligned}
$$

Under consideration of the symmetry of flow field one obtains

$$
\begin{aligned}
\left\langle\xi_{p}{ }^{2}\right\rangle= & \left\langle\eta_{p}{ }^{2}\right\rangle=\frac{1}{3 \mu_{p}} \exp \left\{-\left[t+\left(\dot{\gamma}_{0} \tau_{p} / i \omega\right) e^{i \omega t}\right] / \tau_{p}^{\prime}\right\} \\
& \times \int_{0}^{t / \tau_{p}^{\prime}} \exp \left[x+\left(\dot{\gamma}_{0} \tau_{p} / i \omega \tau_{p}^{\prime}\right) e^{i \omega \tau^{\prime} p} x\right] d x \\
\left\langle\zeta_{p}{ }^{2}\right\rangle= & \frac{1}{3 \mu_{p}} \exp \left\{-\left[t-2\left(\dot{\gamma}_{0} \tau_{p} / i \omega\right) e^{i \omega t}\right] / \tau_{p}^{\prime}\right\} \\
& \times \int_{0}^{t / \tau_{p}^{\prime}} \exp \left[x-2\left(\dot{\gamma}_{0} \tau_{p} / i \omega \tau_{p}^{\prime}\right) e^{i \omega \tau^{\prime} p x}\right] d x \\
\left\langle\xi_{p} \eta_{p}\right\rangle= & \left\langle\eta_{p} \xi_{p}\right\rangle=\left\langle\xi_{p} \zeta_{p}\right\rangle=0 .
\end{aligned}
$$

These averages still contain the transient which, in the general case, cannot be easily separated from the stationary solution reached after the transient has tapered off (fig. 7).

The separation can be performed if $\dot{\gamma}_{0} \tau_{p} / \omega \tau^{\prime}{ }_{p}$ is so small that one can replace the exponential function by its linear expansion. In such a case one obtains for the stationary solution

$$
\begin{aligned}
& \left\langle\xi_{p}{ }^{2}\right\rangle=\frac{1}{3 \mu_{p}}\left(1-\dot{\gamma}_{0} \tau_{p} \frac{e^{i \omega t}}{1+i \omega \tau_{p}^{\prime}}\right) \\
& \left\langle\zeta_{p}{ }^{2}\right\rangle=\frac{1}{3 \mu_{p}}\left(1+2 \dot{\gamma}_{0} \tau_{p} \frac{e^{i \omega t}}{1+i \omega \tau_{p}^{\prime}}\right)
\end{aligned}
$$

The intrinsic Trouton viscosity turns out to be

$[\eta]_{l \omega}^{*}$

$=\left[\sigma_{33}-\sigma_{11}\right] * / \gamma \eta_{s}$

$=\frac{R T}{M \eta_{s}} \sum_{p=1}^{Z} 3 \mu_{p}\left[\left\langle\zeta_{p}{ }^{2}-\xi_{p}{ }^{2}\right\rangle / \dot{\gamma}+\left(\tau_{p} \nu_{p} \varphi_{p} / f\right)\left\langle 2 \zeta_{p}{ }^{2}-\xi_{p}{ }^{2}\right\rangle\right]$

$$
\begin{aligned}
& =\frac{3 R T}{M \eta_{s}} \sum_{p=1}^{Z}\left(\frac{\tau_{p}\left(1-\omega \tau_{p}^{\prime}\right.}{1+\omega^{2} \tau_{p}^{\prime 2}}+\frac{\tau_{p}^{\prime}-\tau_{p}}{3}\right) \\
& =\frac{3 R T}{M \eta_{s}}\left(I / \omega+\sum_{p=1}^{Z}\left(\tau_{p}^{\prime}-\tau_{p}\right) / 3-i \mathrm{II} / \omega\right) .
\end{aligned}
$$

The additional term $\sum\left(\tau_{p}^{\prime}-\tau_{p}\right) / 3$ is independent of $\omega$ and hence represents the Trouton viscosity in the second Newtonian range where $\mathrm{I} / \omega$ and $\mathrm{II} / \omega$ disappear. As in the case of conventional intrinsic viscosity, eq (20), the finite value at $\omega$ $\rightarrow \infty$ is a consequence of internal viscosity, i.e., of partial coil rigidity. The frequency dependence of $[\eta]_{\omega}$ and phase angle $\delta_{l \eta}$ are plotted in figure 8 for $Z=100, h^{*}=.4$, and $\varphi / f=2$.

The intrinsic birefringence reads

$$
[\Delta n]_{l \omega}^{*}=K \frac{R T}{M \eta_{s} \dot{\gamma}} \sum_{p=1}^{Z} 3 \mu_{p}\left\langle\zeta_{p}{ }^{2}-\xi_{p}{ }^{2}\right\rangle
$$

which for small amplitude reduces to

$$
\begin{aligned}
{[\Delta n]_{\omega \omega}^{*} } & =\frac{3 K R T}{M \eta_{s}} \cdot \sum_{p=1}^{Z} \tau_{p} /\left(1+i \omega \tau_{p}^{\prime}\right)=\frac{3 K R T}{M \eta_{s \omega}} \cdot(\mathrm{I}-i \mathrm{II}) \\
\tan \delta_{l n} & =\mathrm{II} / \mathrm{I}
\end{aligned}
$$

This expression differs from the streaming birefringence in an oscillating flow field eq (21), only in the factor 3 . The nonproportionality between viscosity and birefringence is again the consequency of the additional internal viscosity term in eq (33).

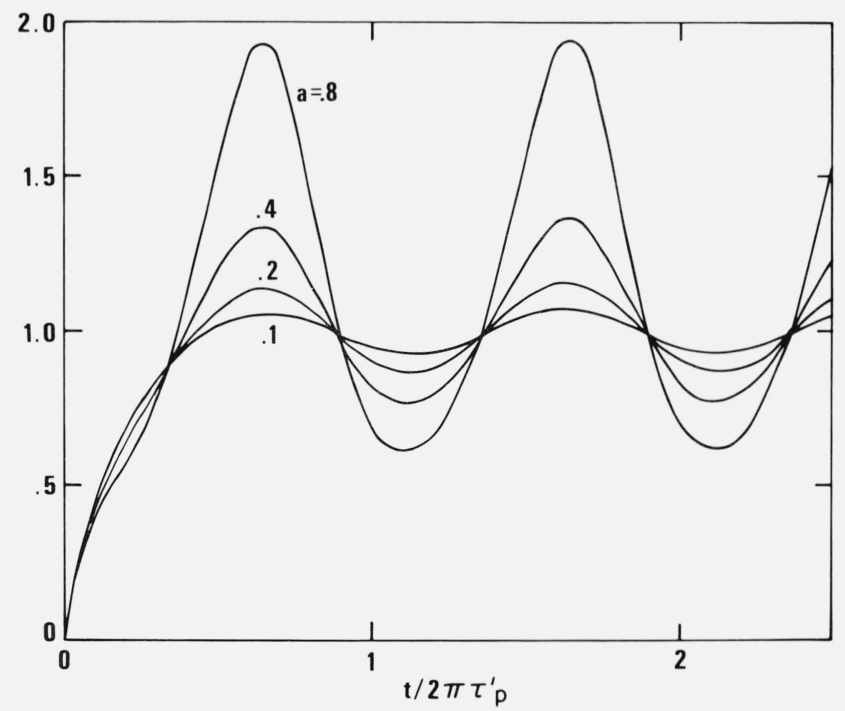

Figure 7. Time dependence of $\left\langle\xi_{\mathrm{p}}{ }^{2}\right\rangle=\left\langle\eta_{\mathrm{p}}{ }^{2}\right\rangle$ according to eq (30) plotted versus $\mathrm{t} / 2 \pi \tau^{\prime}{ }_{\mathrm{p}}$ for the special case $\varphi \tau^{\prime}{ }_{\mathrm{p}}=1$ and $\mathrm{a}=\dot{\gamma}_{0} \tau_{\mathrm{p}} / \omega \tau_{\mathrm{p}}^{\prime}$ showing the short duration of transient effects and the rapid approach to the asymptotic periodic solution.

The stabilization is slower for low $\omega \tau_{p}^{\prime}$. 


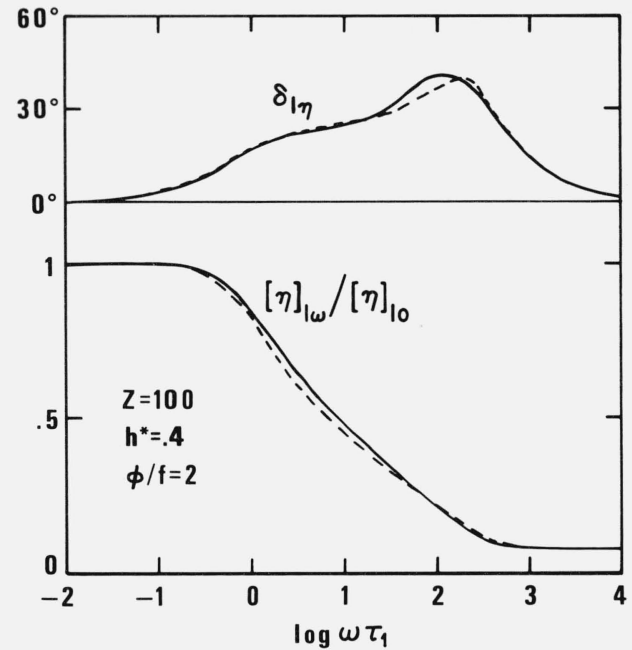

FIGURE 8. Relative intrinsic Trouton viscosity $[\eta]_{l_{\omega}} /[\eta]_{l 0}$ and the phase angle $\delta_{l \eta}$ for $\mathrm{Z}=100, \mathrm{~h}^{*}=.4$, and $\varphi / \mathrm{f}=2$ as function of $\omega \tau_{1}$ according to new (full line) and old (broken line) theory.

Acoustic birefringence is in many respects closely related to birefringence in an oscillating jet flow. The main difference is the absence of lateral contraction as represented by $-\dot{\gamma} x / 2$ and $-\dot{\gamma} y / 2$. In contrast to jet flow with constant specific volume (incompressible liquid) the volume element subjected to an acoustic wave is periodically compressed and expanded. That means a constant $\left\langle\xi_{p}{ }^{2}\right\rangle$ yielding in eq (34) the replacement of the factor 3 by 2 .

One has

$$
\begin{aligned}
{[\Delta n]_{a c}^{*} } & =\lim _{c \rightarrow 0} \frac{\Delta \mathrm{n}}{n c \mathrm{I}_{a c}^{1 / 2}} \\
& =\frac{N}{M} K_{a c} \frac{\omega \tau}{\left(1+\omega^{2} \tau^{2}\right)^{1 / 2}} \sin \left[\omega\left(t-z_{0} / c_{a}\right)-\delta_{a c}\right] \\
& =\frac{N}{M} \cdot K_{a c} \cdot \sum_{p=1}^{Z} \frac{-i \omega \tau_{p}}{1+i \omega \tau_{p}^{\prime}} e^{i \omega\left(t-z_{0} / c_{a}\right)} \\
& =\frac{N}{M} K_{a c}(\mathrm{I}-i \mathrm{II}) e^{i \omega\left(t-z_{0} / c_{a}\right)}
\end{aligned}
$$

$$
\begin{aligned}
\tan \delta_{a c} & =\mathrm{II} / \mathrm{I} \\
K_{a c} & =\frac{4 \pi}{5}\left(\frac{n^{2}+2}{3 n}\right)^{2}\left(\frac{2}{\rho_{c^{3} a c}}\right)^{1 / 2}\left(\alpha_{1}-\alpha_{2}\right) \\
\mathrm{I}_{a c} & =\rho B^{2} c_{a c} / 2 .
\end{aligned}
$$

Here $z_{0}$ is the location of the center of hydrodynamic resistance of the macromolecule, $\mathrm{B}$ is amplitude, $\mathrm{I}_{a c}$ is intensity and $c_{a c}$ is propagation velocity of acoustic wave, and $\rho$ is the density of solution. The frequency dependence of acoustic birefringence and phase angle is exactly the same as in the case of oscillating jet flow.
The difference between acoustic birefringence according to the correct new and the incorrect old values $\nu_{p}$ can be seen in figure 3 where $[\Delta n]_{a c}$ is plotted versus $\omega \tau_{1}$ for $Z=100, h^{*}=$ .4 and $\varphi / f=2$. The phase angle $\delta_{a c}$ is identical with $\delta_{n}$ and $\delta_{l n}$.

\section{Conclusions}

The paper presents the calculation of most of the intrinsic rheological and rheooptical effects of linear homopolymers in an oscillating flow field which may be explored experimentally. In the case of rheooptical effects only the intrinsic birefringence of the polymer is included. The form-birefringence is completely neglected. The same applies to the influence of large side groups which may effect independently the optical anisotropy of the segment and its frequency dependence.

The introduction of the appropriate $\nu_{p}=1$ values instead of the old values $\nu_{p}=\lambda_{p Z} / \lambda_{p R}$ does not change drastically the effects depending on internal viscosity. As a rule the ratio between the new and old values of intrinsic viscosity, birefringence, and first normal stress difference is less than 2 , at least in the range of $Z$ between 3 and 300 . With higher $Z$ the differences increase as a linear function of $\log Z$.

As already mentioned, the smallness of the difference is a consequence of the peculiar dependence of old $\nu^{p(1)}$ on $p$ : much larger than 1 at small $p$ and smaller than 1 at high $p$. Hence the larger contributions in the former part of the sums are partially compensated by the smaller contributions in the latter part.

The most important changes occur in the second Newtonian intrinsic viscosity which is the most conspicuous consequence of internal viscosity. Here the dependence of $[\eta]_{\infty}$ on $Z$ is much less according to the new theory than it was in the case of the old theory.

No calculation of the gradient effects was attempted because one knows that in a flow with a finite velocity gradient the randomly coiled macromolecule is deformed with a consequent change of all interbead distances. This yields a change of interaction tensor $\mathbf{H}$ which leads to a modification of all eigenvalues $\lambda_{p}$. The rest values $\lambda_{p}$ used in this paper are only applicable to effects where an extrapolation to zero gradient is straightforward. This is the case with dynamic effects where one uses very small gradients and concentrates on the frequency dependence of the effects measured. The situation, however, is basically different in the non-linear range of gradient dependence of excess stress and optical tensor.

\section{References}

[1] Fong, J. T., and Peterlin, A., J. Research NBS, Section B, Mathematical Sci., 80, 273 (1976)

[2] Zimm, B. H., J. Chem. Phys. 24, 269 (1956).

[3] Zimm, B. H., Roe, G. M., and Epstein, L. F., J. Chem. Phys. 24, 279 (1956).

[4] Hearst, J. E., J. Chem. Phys. 37, 2547 (1962).

[5] Pyun, C. W., and Fixman, M. J., J. Chem. Phys. 42, 3838 (1965).

[6] Thurston, G. B., and Morrison, J. D., Polymer 10, 427 (1969).

[7] Lodge, A. S., and Wu. Y.. Rheol. Acta 10, 539. (1971).

[8] Lodge, A. S. and Wu, Y., University of Wisconsin, Mathematics Research Center, Report No 1250, Madison, W isconsin (1972).

[9] Rouse, P. E., J. Chem. Phys. 21, 1272 (1953).

[10] Cerf, R., J. Phys. Radium 19, 122 (1958).

[11] Cerf, R., Adv. Polymer Sci. 1, 382 (1959).

[12] Chaffey, C., J. Chem. Phys. 63, 1385 (1966). 
[13] Peterlin, A., Kolloid Z. and Z. Polymere 209, 181 (1966).

[14] Peterlin, A., J. Polvmer Sci. A2, 5, 179 (1967).

[15] Peterlin, A., and Reinhold, C., Trans. Soc. Rheol. 11 (1) 15 (1967).

[16] Peterlin, A.. J. Polvmer Sci. B5, 113 (1967).

[17] Peterlin, A., Polymer 8, 21 (1967).

[18] Thurston, G. B., and Peterlin, A., J. Chem. Phys. 46, 4881 (1967).

[19] Peterlin, A., Non Newtonian Viscosity and the Macromolecule, Adv. Macromol. Chem. 1, 225 (1968).

[20] Peterlin, A., J. Polymer Sci. B10, 101 (1972).

[21] Peterlin, A., Viscosity of Polymers, "Physical Chemistry" Ser. 1, Vol. 8, Edited by C. E. M. Bawn (Butterworth-University Park Press, London-Baltimore, 1972), p. 199.

[22] Peterlin, A., and Munk, P., Streaming Birefringence, Physical Methods of Chemistry, Edited by A. Weisberger and B. Rossiter (J. Wiley \& Sons, N. Y., 1972, Vol. 1, Part III), p. 271.

[23] Peterlin, A., J. Polvmer Sci. C43, 187 (1973).

[24] Peterlin, A., Rheol. Acta 12, 496 (1973).

[25] Cerf, R., Chem. Phys. Lett. 24, 317 (1974).

[26] Cerf, R., Adv. Chem. Phys. 33, 73 (1975).

[27] Peterlin, A., and Copič, M., J. Appl. Phys. 27, 434 (1956).

[28] Booij, H. C., and van Wiechen, P. H., J. Chem. Phys. 52, 5056 (1970).

[29] Fixman, M., and Stockmayer, W. H., Am. Rev. Phys. Chem. 21, 407 (1970).

[30] Osaki, K., Adv. Polymer Sci. 12, 1 (1973).

[31] Kuhn, W., and Kuhn, H., Helv. Chim. Acta 28, 1533 (1945).

[32] Kuhn, W., and Kuhn, H., Helv. Chim. Acta 29, 72 (1946).

[33] See for instance the review articles (Ref. [29]), and Stockmayer, W. H., Polymer Conformation and Dynamics in Solution, Ann. Rev. Phys. Chem. 21, 407, (1970); Yamakawa, M., Polymer Mechanics, Ann. Rev. Phys. Chem. 25, 179 (1974).

[34] Edwards, S. F., and Freed, K. F., J. Chem. Phys. 61, 1189 (1974).

[35] Felderhof, B. V., Deutch, J. M., and Titulear, U. M., J. Chem. Phys. 63, 740 (1975).
[36] Tschoegl, N. W., and Ferry, J. D., Kolloid Z \& Z. Polymer 189, 37 (1963).

[37] Philippoff, W., Trans. Soc. Rheol. 8, 117 (1964).

[38] Lamb, J., and Matheson, A. J., Proc. Roy. Soc. (London), A281, 207 (1964).

[39] Tschoegl, N. W., and Ferry, J. D., J. Phys. Chem. 68, 867 (1964).

[40] Harrison, J., Lamb, J., and Matheson, A. J., J. Phys. Chem. 68, 1072 , (1964).

[41] Frederick, J. E., Tschoegl, N. W., and Ferry, J. D., J. Phys. Chem. 68, 1974 (1964).

[42] Frederick, J. E., and Ferry, J. D., J. Phys. Chem. 69, 346 (1965).

[43] Ferry, J. D., Holmes, L. A., Lamb, J., and Matheson, A. J., J. Phys. Chem. 70, 1685 (1966).

[44] Massa, D. J., Schrag, L. J., and Ferry, J. D., Macromol. 4, 210 (1971).

[45] Osaki, K., and Schrag, J. L., Polvmer J. (Japan) 2, 541 (1971).

[46] Holmes, L. A., Kusamizu, S., Osaki, K., and Ferry, J. D., J. Polymer Sci. A2, 9, 2009 (1971).

[47] Massa, D. J., and Schrag, J. L., J. Polymer Sci. A2, 10, 71, (1972).

[48] Osaki, K., Mitsuda, Y., Johnson, R. M., Schrag, J. L., and Ferry, J. D., Macromol. 5, 17 (1972).

[49] Osaki, K., and Schrag, J. L., J. Polymer Sci. (Physics Edit.) 11, 549 (1973).

[50] Mitsuda, Y., Osaki, J., Schrag, J. L., and Ferry, J. D., Polymer J. (Japan) 4, 24 (1973).

[51] Noordermeer, J. W. M., Kramer, O., Nestler, F. H. M., Schrag, J. L., and Ferry, J. D., Macromol. 8, 539 (1975).

[52] Peterlin, A., and Stuart, H. A., Hd. \& Jbch. Chem. Phys. 8/1B (Akad. Verlagsges. Leipzig, 1943).

[53] Leray, J., J. Polymer Sci. 23, 167 (1957).

a) US GOVERNMENT PRINTING OFFICE: 1977 - 241-306/1 